\title{
Successful Treatment of a Panniculitis-Like Primary Cutaneous T-Cell Lymphoma of the $\alpha / \beta$ Type with Bexarotene
}

\author{
X. Zhang M. Schlaak M. Fabri C. Mauch P. Kurschat \\ University Hospital of Cologne, Department of Dermatology, Skin Cancer \\ Centre, Cologne, Germany
}

\section{Key Words}

Cutaneous T-cell lymphoma - Lymphoma Panniculitis · Therapy · Retinoids · Bexarotene

\begin{abstract}
Subcutaneous panniculitis-like T-cell lymphoma (SPTL) of the $\alpha / \beta$ type is a rare subtype of non-Hodgkin's lymphoma of the skin. Although these tumors usually run an indolent course, disease-related morbidity is often severe. Clinical findings include subcutaneous tumors located on the extremities or trunk, often accompanied by systemic symptoms like fever or fatigue. Due to the low incidence of SPTL, no standardized therapy has been defined so far and there is currently no curative therapy available for this type of non-Hodgkin's lymphoma. By sharing our experience with bexarotene therapy, we present a safe and potentially improved treatment for patients with SPTL. In the case presented, bexarotene was able to induce remission even after recurrence of disease.
\end{abstract}

\section{Introduction}

Subcutaneous panniculitis-like T-cell lymphoma (SPTL) is a rare subgroup of primary cutaneous T-cell lymphoma with major manifestation in the subcutaneous tissues [1]. Histologically, SPTL is characterized by infiltration of pleomorphic T cells in the subcutis, mimicking lobular panniculitis [2,3]. Given clear differences in disease outcome the World Health Organization-European Organization for Research and Treatment of Cancer (WHO-EORTC) classification for cutaneous lymphomas defines as SPTL only cases with an $\alpha / \beta+$ T-cell phenotype and a usually indolent clinical course. In contrast, the aggressive $\gamma / \delta+$ form of cutaneous T-cell lymphomas with frequent subcutaneous involvement was separated as a distinct entity [2]. We describe complete 
disease remission in a 63-year-old woman with primary cutaneous $\alpha / \beta$-type SPTL following treatment with bexarotene and prednisone. In light of a lack of standard treatment for SPTL, we describe our experiences with using bexarotene as a first-line therapy and its efficacy in $\alpha / \beta$-type SPTL.

\section{Case Report}

A 63-year-old woman presented with painful subcutaneous nodules bilaterally on her upper thighs (fig. 1a). She had B-type symptoms of fever and night sweats. Histologically, a first biopsy specimen was diagnosed as lobular panniculitis. The patient had a history of tachycardiac arrhythmias, mild depression and hyperlipidemia. No other diseases were known. Without evidence of pancreatitis, alpha 1-antitrypsin deficiency, or lupus erythematosus, the lesions were initially diagnosed as idiopathic panniculitis. Initial treatment with systemic prednisone of up to $100 \mathrm{mg}$ per day and NSAIDs like ibuprofen led to some improvement without remission. Additional histopathology taken 8 months after onset of disease revealed dense lobular lymphocytic infiltrates rimming around adipocytes (fig. 2a, b) with the infiltrates staining positively for CD3, CD8 (fig. 2c, d), perforin, TIA-1, and negatively for CD4, CD5, CD56. Complete staging, including CT of thorax and abdomen and head MRI revealed no systemic involvement. No infiltration was found in bone marrow biopsy. T-cell receptor polymerase chain reaction (PCR) from skin biopsy was performed by a reference laboratory and revealed clonality as well as an $\alpha / \beta$ phenotype. The combination of clinical findings, histopathology and molecular biology prompted us to make the diagnosis of primary $\alpha / \beta$ SPTL.

The patient was started on a treatment of $150 \mathrm{mg} /$ day bexarotene and $80 \mathrm{mg} /$ day prednisone. Bexarotene was increased to $300 \mathrm{mg} /$ day while prednisone was tapered in step-wise fashion. Clinically, a rapid improvement was observed after initiation of treatment (fig. 1b). After 14 months complete remission was achieved and bexarotene was discontinued. In the following 2 years two recurrences occurred. For the first recurrence, bexarotene alone was given for 4 months, after which a complete remission was seen. Bexarotene alone was again reintroduced for the second recurrence and clinical improvement but no complete remission is currently observed after a follow up of 5 months.

\section{Discussion}

SPTL was first described in 1991 by Gonzalez et al. as a distinct form of cutaneous lymphoma characterized by subcutaneous tissue localization, aggressive clinical course and frequent association with hemophagocytic syndrome [4]. Clinical manifestations involve multiple nodular skin lesions or plaques on the extremities or trunk. Systemic symptoms such as fever, weight loss and fatigue may also be present. There are two distinct types of SPTL with very different disease outcome. In a study of 83 cases of SPTL, patients with $\alpha / \beta+T$-cell phenotype had an indolent clinical course with a fiveyear survival of $82 \%$ [1]. In the same study, patients with $\gamma / \delta+T$-cell phenotype showed a more aggressive disease with extracutaneous dissemination, and the fiveyear survival was $11 \%$. This clear difference in clinical outcome necessitates development of separate therapeutic strategies for each type of SPTL.

Bexarotene is a retinoid that selectively activates the retinoid X receptors (RXR) [5]. In a clinical trial with heavily pretreated refractory cutaneous T-cell lymphoma (CTCL) patients, bexarotene treatment resulted in a response rate of $54 \%$ in early-stage CTCL and $45 \%$ in advanced-stage CTCL patients [6]. Side effects of bexarotene treatment include elevation in lipid and cholesterol levels and hypothyroidism at higher doses. Thus, patients on bexarotene need to be closely monitored for toxicity, and lipidlowering medications and levothyroxine might be necessary. 
Currently there is no standardized therapy for $\alpha / \beta$ SPTL. A number of treatment strategies including chemotherapy, systemic corticosteroid therapy, local radiotherapy and surgery have been reported with various success rates [7-10]. In a study of 63 patients with $\alpha / \beta$ SPTL, 31 patients were treated with cyclophosphamide, doxorubicin, vincristine, and prednisone (CHOP) or similar chemotherapy and 19 patients showed complete remission. Sixteen of 24 patients went into complete remission after they were given less aggressive initial treatments, including combinations of prednisone, chlorambucil, methotrexate, cyclophosphamide, interferon- $\alpha$, and gemcitabine [1]. However, no chemotherapeutic regimen tried so far has been able to cure SPTL.

We present the first report of complete remission in a SPTL patient with combined bexarotene and prednisone treatment. It is not possible to clearly assess the contribution of each substance to the therapeutic effect. But the fact that a prior therapy with prednisone and NSAIDs did not result in complete remission substantiates the contribution of bexarotene. Furthermore, after discontinuation of treatment, the patient presented with a relapse of SPTL, but went into remission when monotherapy with bexarotene was restarted. In comparison to the dosage used to treat mycosis fungoides, the patient showed remission with relatively low daily doses of 300 $\mathrm{mg} /$ day of bexarotene. Studies have demonstrated that bexarotene is generally well tolerated at the lower doses (150-300 mg/day), which results in limited toxicity [11]. Low toxicity makes bexarotene a less aggressive and, taking into consideration the indolent nature of SPTL, potentially a better initial therapy, as compared to CHOP or methotrexate. Patients with SPTL are known to suffer numerous relapses after establishing complete remission. For patients who tolerate bexarotene well, continuous low-dose treatment might be utilized after complete remission to maintain the diseasefree state.

\section{Disclosure Statement}

The authors have no conflict of interest to disclose. 

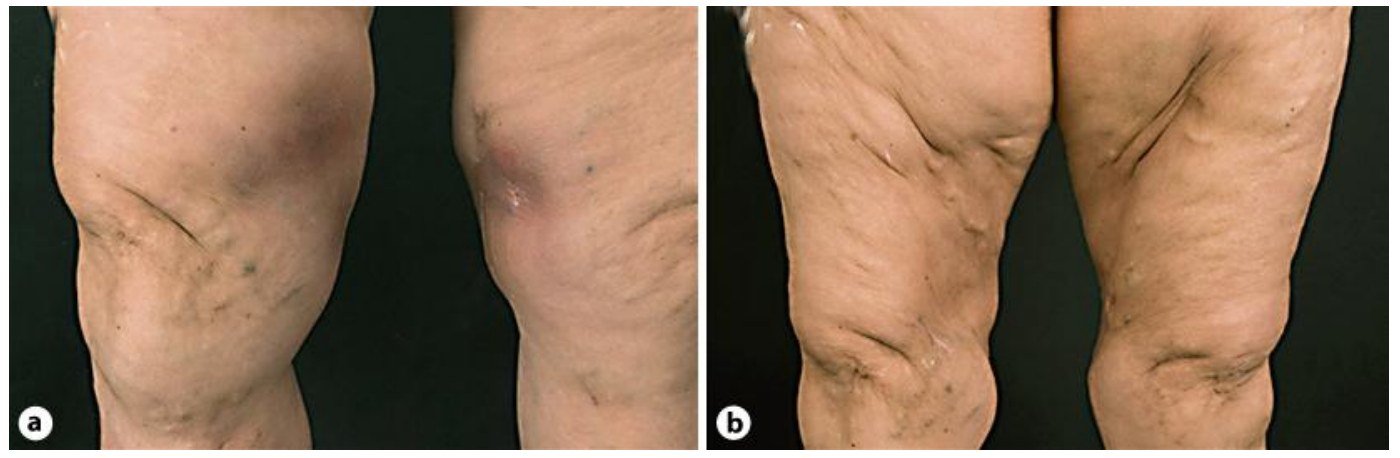

Fig. 1. a On admission painful erythematous plaques were observed on the upper medial thighs bilaterally. $\mathbf{b}$ After seven months of treatment with bexarotene and prednisone, infiltration and reddening of the skin were no longer present, leaving atrophy of the subcutaneous tissue.
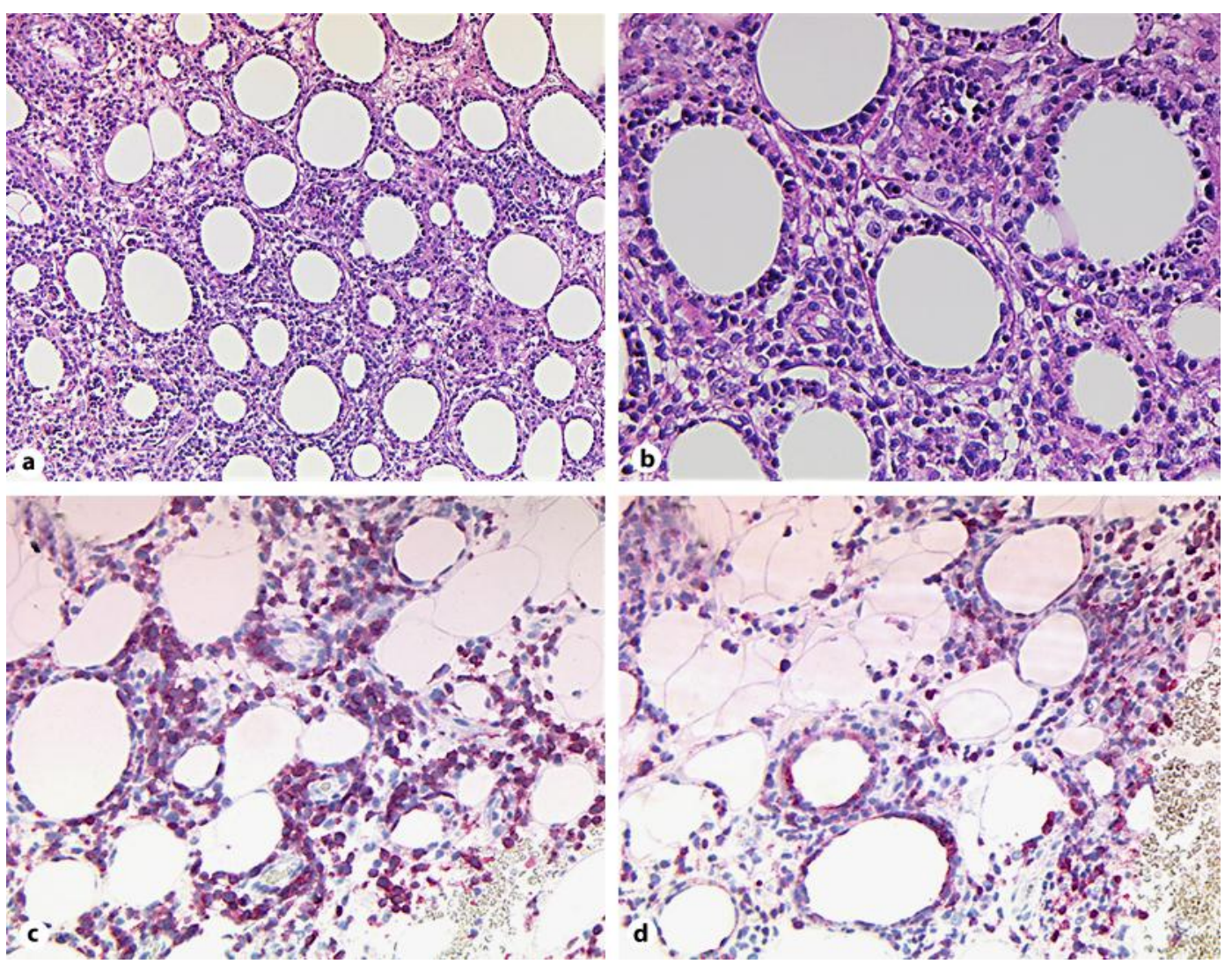

Fig. 2. a, b H\&E staining of lesional skin reveals dense lymphocytic infiltration of adipose tissue with classical rimming of atypical lymphocytes around adipocytes. $\mathbf{c}, \mathbf{d}$ The infiltrates were determined to be cytotoxic lymphocytes with positive immunohistochemical staining for CD3 and CD8, respectively. 


\section{References}

1 Willemze R, Jansen PM, Cerroni L, Berti E, Santucci M, Assaf C, Canninga-van Dijk MR, Carlotti A, Geerts ML, Hahtola S, Hummel M, Jeskanen L, Kempf W, Massone C, Ortiz-Romero PL, Paulli M, Petrella T, Ranki A, Peralto JL, Robson A, Senff NJ, Vermeer MH, Wechsler J, Whittaker S, Meijer CJ: Subcutaneous panniculitis-like T-cell lymphoma: definition, classification, and prognostic factors: an EORTC Cutaneous Lymphoma Group Study of 83 cases. Blood 2008;111:838-845.

-2 Willemze R, Jaffe ES, Burg G, Cerroni L, Berti E, Swerdlow SH, Ralfkiaer E, Chimenti S, Diaz-Perez JL, Duncan LM, Grange F, Harris NL, Kempf W, Kerl H, Kurrer M, Knobler R, Pimpinelli N, Sander C, Santucci M, Sterry W, Vermeer MH, Wechsler J, Whittaker S, Meijer CJ: WHO-EORTC classification for cutaneous lymphomas. Blood 2005;105:3768-3785.

-3 Jaffe ES: The 2008 WHO classification of lymphomas: implications for clinical practice and translational research. Hematology Am Soc Hematol Educ Program 2009:523-531.

-4 Gonzalez CL, Medeiros LJ, Braziel RM, Jaffe ES: T-cell lymphoma involving subcutaneous tissue. A clinicopathologic entity commonly associated with hemophagocytic syndrome. Am J Surg Pathol 1991;15:17-27.

-5 Boehm MF, Zhang L, Badea BA, White SK, Mais DE, Berger E, Suto CM, Goldman ME, Heyman RA: Synthesis and structure-activity relationships of novel retinoid $\mathrm{X}$ receptor-selective retinoids. J Med Chem 1994;37:2930-2941.

-6 Duvic M, Hymes K, Heald P, Breneman D, Martin AG, Myskowski P, Crowley C, Yocum RC: Bexarotene is effective and safe for treatment of refractory advanced-stage cutaneous T-cell lymphoma: multinational phase II-III trial results. J Clin Oncol 2001;19:2456-2471.

-7 Hashimoto H, Sawada K, Koizumi K, Nishio M, Endo T, Takashima T, Kobayashi H, Koike T: Effective CD34+ selected autologous peripheral blood stem cell transplantation in a patient with subcutaneous panniculitic T cell lymphoma (SPTCL) transformed into leukemia. Bone Marrow Transplant 1999;24:1369-1371.

8 Haycox CL, Back AL, Raugi GJ, Piepkorn M: Subcutaneous T-cell lymphoma treated with systemic chemotherapy, autologous stem cell support, and limb amputation. J Am Acad Dermatol 1997;37:832 835 .

-9 Briki H, Bouaziz JD, Molinier-Frenkel V, Delfau-Larue MH, Ortonne N, Bagot M: Subcutaneous panniculitis-like T-cell lymphoma $\alpha \beta$ : complete sustained remission with corticosteroids and methotrexate. Br J Dermatol 2010;163:1136-1138.

10 Hathaway T, Subtil A, Kuo P, Foss F: Efficacy of denileukin diftitox in subcutaneous panniculitis-like Tcell lymphoma. Clin Lymphoma Myeloma 2007;7:541-545.

-11 Miller VA, Benedetti FM, Rigas JR, Verret AL, Pfister DG, Straus D, Kris MG, Crisp M, Heyman R, Loewen GR, Truglia JA, Warrell RP Jr: Initial clinical trial of a selective retinoid X receptor ligand, LGD1069. J Clin Oncol 1997;15:790-795. 